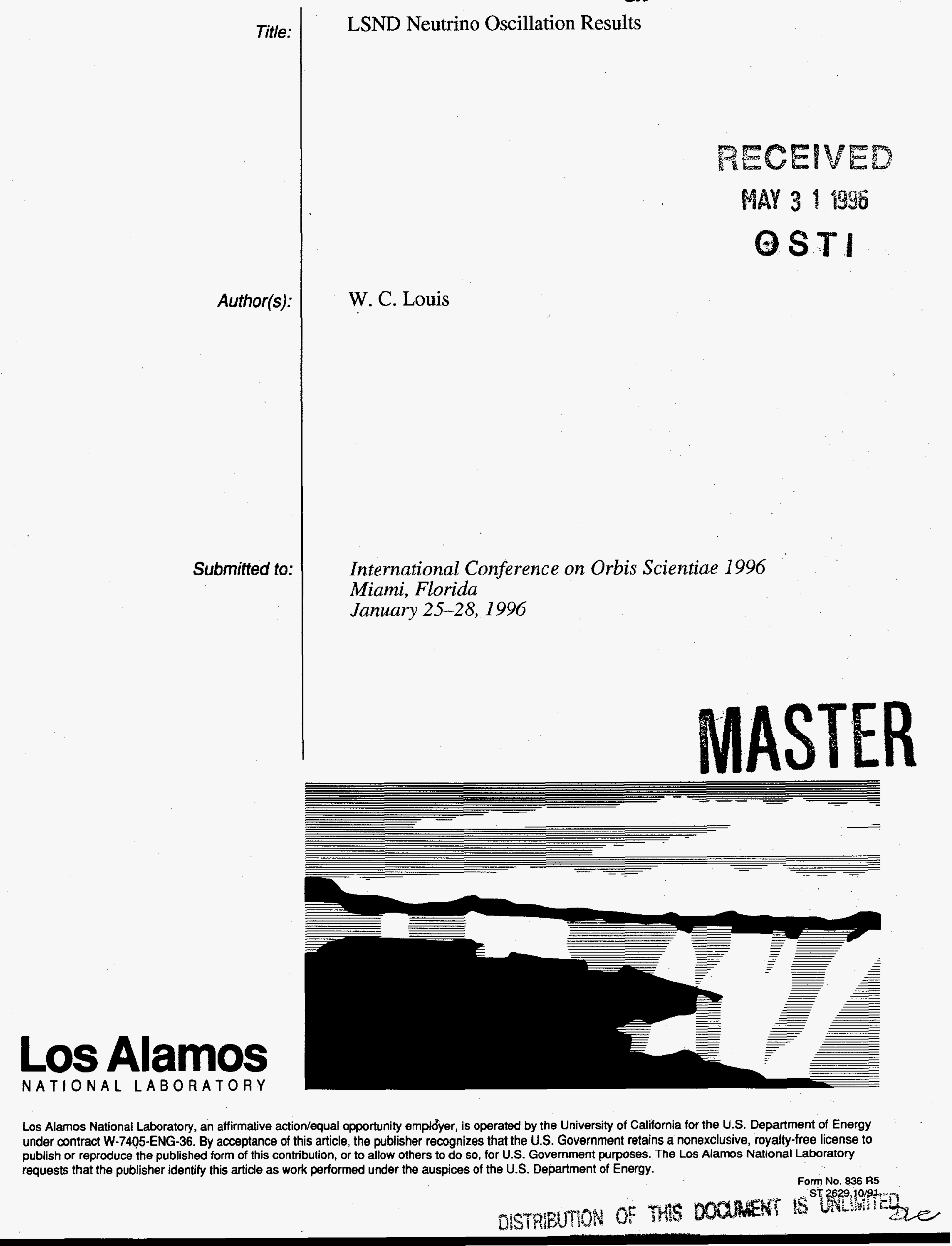




\section{DISCLAMMER}

Portions of this document may be illegible in electronic image products. Images are produced from the best available original document. 


\title{
LSND NEUTRINO OSCILLATION RESULTS
}

\author{
W. C. Louis, representing the LSND Collaboration ${ }^{1}$ \\ Los Alamos National Laboratory \\ Physics Division \\ Los Alamos, NM 87545, U.S.A.
}

\section{INTRODUCTION}

In the past several years, a number of experiments have searched for neutrino oscillations, where a neutrino of one type (say $\vec{\nu}_{\mu}$ ) spontaneously transforms into a neutrino of another type (say $\bar{\nu}_{e}$ ). For this phenomenon to occur, neutrinos must be massive and the apparent conservation law of lepton families must be violated. In 1995 the LSND experiment published data showing candidate events that are consistent with $\bar{\nu}_{\mu} \rightarrow \bar{\nu}_{e}$ oscillations. ${ }^{2}$ Additional data are reported here which provide stronger evidence for neutrino oscillations. ${ }^{3}$

\section{THE LSND EXPERIMENT}

The Liquid Scintillator Neutrino Detector (LSND) experiment at LAMPF ${ }^{3}$ is designed to search with high sensitivity for $\bar{\nu}_{\mu} \rightarrow \bar{\nu}_{e}$ oscillations from $\mu^{+}$decay at rest. LAMPF is a most intense source of low energy neutrinos due to its 1 $\mathrm{mA}$ proton intensity and $800 \mathrm{MeV}$ energy. The neutrino source is well understood because almost all neutrinos arise from $\pi^{+}$or $\mu^{+}$decay; $\pi^{-}$and $\mu^{-}$are readily captured in the $\mathrm{Fe}$ of the shielding and $\mathrm{Cu}$ of the beam stop. The production of kaons and heavier mesons is negligible at these energies. The $\bar{\nu}_{e}$ rate is calculated to be only $4 \times 10^{-4}$ relative to $\bar{\nu}_{\mu}$ in the $36<E_{\nu}<52.8 \mathrm{MeV}$ energy range, so that the observation of a significant $\bar{\nu}_{e}$ rate would be evidence for $\bar{\nu}_{\mu} \rightarrow \bar{\nu}_{e}$ oscillations.

The LSND detector consists of an approximately cylindrical tank $8.3 \mathrm{~m}$ long by $5.7 \mathrm{~m}$ in diameter. The center of the detector is $30 \mathrm{~m}$ from the neutrino source. On the inside surface of the tank 1220 8-inch Hammamatsu phototubes provide $25 \%$ photocathode coverage. A schematic of the LSND detector is shown in Fig. 1. The tank is filled with 167 metric tons of liquid scintillator consisting of mineral oil and $0.031 \mathrm{~g} / 1$ of b-PBD. The low scintillator concentration allows the detection of both Cerenkov light and scintillation light and yields a relatively long attenuation length of more than $20 \mathrm{~m}$ for wavelengths greater than $400 \mathrm{~nm} .{ }^{4}$ A typical $45 \mathrm{MeV}$ electron created in the detector produces a total of $\sim 1500$ photoelectrons, of which $\sim 280$ 
photoelectrons are in the Cerenkov cone. The phototube time and pulse height signals are used to reconstruct the track with an average r.m.s. position resolution of $\sim 30 \mathrm{~cm}$, an angular resolution of $\sim 12$ degrees, and an energy resolution of $\sim 7 \%$. The Cerenkov cone for relativistic particles and the time distribution of the light, which is broader for non-relativistic particles, give excellent particle identification. Surrounding the detector is a veto shield ${ }^{5}$ which tags cosmic ray muons going through the detector.

\section{DATA}

The signature for a $\bar{\nu}_{e}$ interaction in the detector is the reaction $\bar{\nu}_{e} p \rightarrow e^{+} n$ followed by $n p \rightarrow d \gamma(2.2 \mathrm{MeV})$. A likelihood ratio, $\mathrm{R}$, is employed to determine whether a $\gamma$ is a $2.2 \mathrm{MeV}$ photon correlated with a positron or is from an accidental coincidence. $\mathrm{R}$ is the likelihood that the $\gamma$ is correlated, divided by the likelihood that it is accidental. $\mathrm{R}$ depends on the number of hit phototubes for the $\gamma$, the reconstructed distance between the positron and the $\gamma$, and the relative time between the $\gamma$ and positron. Fig. 2 shows the expected $\mathrm{R}$ distribution for accidental photons and correlated photons. Fig. 3 shows the $\mathrm{R}$ distribution, beam on minus beam off, for events with positrons in the $36<E<60 \mathrm{MeV}$ energy range. The dashed histogram is the result of the $\mathrm{R}$ fit for events without a recoil neutron, and the solid histogram is the total fit, including events with a neutron. After subtracting the neutrino background with a recoil neutron there is a total excess of $54.8_{-15.8}^{+18.5} \pm 8.2$ events, which if due to neutrino oscillations corresponds to an oscillation probability of $\left(0.33_{-0.10}^{+0.11} \pm 0.05\right) \%$.

Fig. 4 shows the electron energy distribution, beam on minus beam off excess, for events (a) without a $\gamma$ requirement and (b) events with an associated $\gamma$ with $R>30$. For this latter requirement, the total $2.2 \mathrm{MeV} \gamma$ detection efficiency is $23 \%$ and the probability that an event has an accidental $\gamma$ in coincidence is $0.6 \%$. The dashed histogram shows the background from expected neutrino interactions. There are 22 events beam on in the $36<E<60 \mathrm{MeV}$ energy range and a total estimated background (beam off plus neutrino-induced background) of $4.6 \pm 0.6$ events. Table 1 lists the properties of these 22 events, while Table 2 shows the background estimate for events in the $36<E_{e}<60 \mathrm{MeV}$ energy range with $R \geq 0$ and $R>30$. Fig. 5 shows the spatial distributions for the beam on-off excess events with $R \geq 0$ and $R>30$. The probability that this excess is due to a statistical fluctuation is $<10^{-7}$. If the observed excess is due to neutrino oscillations, Fig. 6 shows the allowed region ( $90 \%$ and $99 \%$ likelihood regions) of $\sin ^{2} 2 \theta$ vs $\Delta m^{2}$ from a maximum likelihood fit to the $\mathrm{L} / \mathrm{E}$ distribution of the 22 beam on events. Some of the allowed region is excluded by the ongoing KARMEN experiment at ISIS, ${ }^{6}$ the E776 experiment at BNL, ${ }^{7}$ and the Bugey reactor experiment. ${ }^{8}$

\section{CONCLUSION}


In summary, the LSND experiment observes an excess of events with positrons in the $36<E<60 \mathrm{MeV}$ energy range that are correlated in time and space with a low energy $\gamma$. If the observed excess is interpreted as $\bar{\nu}_{\mu} \rightarrow \bar{\nu}_{e}$ oscillations, it corresponds to an oscillation probability of $\left(0.33_{-0.10}^{+0.11} \pm 0.05 \%\right)$ for the allowed regions shown in Fig. 6. More data taking is planned for the experiment, and the performance of the detector is under continuous study. Both of these efforts are expected to improve the understanding of the phenomena described here. If neutrino oscillations have in fact been observed, then the minimal standard model would need to be modified and neutrinos would have mass sufficient to influence cosmology and the evolution of the universe.

\section{REFERENCES}

${ }^{1}$ The LSND Collaboration consists of the following people and institutions: $K$. McIlhany, I. Stancu, W. Strossman, G. J. VanDalen (Univ. of California, Riverside); W. Vernon (Univ. of California, San Diego and IIRPA); D. O. Caldwell, M. Gray, S. Yellin (Univ. of California, Santa Barbara); D. Smith, J, Waltz (Embry-Riddle Aeronautical Univ.); A. M. Eisner, Y-X. Wang (Univ. of California IIRPA); I. Cohen (Linfield College); R. L. Burman, J. B. Donahue, F. J. Federspiel, G. T. Garvey, W. C. Louis, G. B. Mills, V. Sandberg, R. Tayloe, D. H. White (Los Alamos National Laboratory); R. M. Gunasingha, R. Imlay, H. J. Kim, W. Metcalf (Louisiana State Univ.): K. Johnston (Louisiana Tech Univ.); B. D. Dieterle, R. A. Reeder (Univ. of New Mexico); A. Fazely (Southern Univ); C. Athnassopoulos, L. B. Auerbach, R. Majkic, J. Margulies, D. Works, Y. Xiao (Temple Univ.).

${ }^{2}$ C. Athanassopoulos et. al. , Phys. Rev. Lett. 75, 2650 (1995).

${ }^{3}$ C. Athanassopoulos et. al., submitted to Phys. Rev. C.

${ }^{4}$ R. A. Reeder et. al. , Nucl. Instrum. Methods A 334, 353 (1993).

${ }^{5}$ J. J. Napolitano et. al. , Nucl. Instrum. Methods A 274, 152 (1989).

${ }^{6}$ B. Bodmann et. al. , Phys. Lett. B 267, 321 (1991); B. Bodmann et. al. , Phys. Lett. B 280, 198 (1992); B. Zeitnitz et. al. , Prog. Part. Nucl. Phys., 32351 (1994).

${ }^{7}$ L. Borodovsky et. al. , Phys. Rev. Lett. 68, 274 (1992).

${ }^{8}$ B. Achkar et. al., Nucl. Phys. B434, 503 (1995).

\section{DISCLAIMER}

account of work sponsored by an agency of the United States This report was prepared as an account of work sponsored any agency thereof, nor any of their Government. Neither the United States Goverimplied, or assumes any legal liability or respons, emplayees, makes any warranty, express or implies, of any information, apparatus, product, bility for the accuracy, completeness, or use would not infringe privately owned rights. Referprocess disclosed, or represents that is use woct, process, or service by trade name, traderemence herein to any specific commercial processarily constitute or imply its endorsement, The views manufacturer, or otherwise does not necessarily constion or and any agency thereof. The views
mendation, or favoring by the United States Government or any

and opinions of authors expressed herein do thereof. 
TABLE I. The 22 beam-on events with $R>30$ and energy in the $36<E_{e}<60 \mathrm{MeV}$ range. For each event is given year recorded, energy, spatial position, and distance from the PMT surfaces.

\begin{tabular}{|c|c|c|c|c|c|c|}
\hline Event & Year & $\mathrm{E}(\mathrm{MeV})$ & $\mathrm{X}(\mathrm{cm})$ & $\mathrm{Y}(\mathrm{cm})$ & $\mathrm{Z}(\mathrm{cm})$ & $D(\mathrm{~cm})$ \\
\hline 1 & 1993 & 47.6 & -66 & -84 & -77 & 115 \\
\hline 2 & 1993 & 51.1 & 56 & -96 & 53 & 103 \\
\hline 3 & 1994 & 40.1 & -36 & 196 & -203 & 53 \\
\hline 4 & 1994 & 44.2 & 69 & -146 & 153 & 53 \\
\hline 5 & 1994 & 39.4 & -169 & 96 & -347 & 39 \\
\hline 6 & 1994 & 36.3 & -156 & -79 & -207 & 84 \\
\hline 7 & 1994 & 52.9 & 21 & 106 & 71 & 143 \\
\hline 8 & 1994 & 37.0 & 31 & 156 & -105 & 93 \\
\hline 9 & 1994 & 42.4 & -14 & -121 & .239 & 78 \\
\hline 10 & 1994 & 37.7 & -91 & 119 & 209 & 109 \\
\hline 11 & 1994 & 54.3 & -91 & 191 & 269 & 47 \\
\hline 12 & 1994 & 55.8 & 71 & -99 & -259 & 100 \\
\hline 13 & 1994 & 43.8 & 6 & 211 & 173 & 38 \\
\hline 14 & 1995 & 49.2 & -184 & 10 & 58 & 75 \\
\hline 15 & 1995 & 56.5 & 128 & -150 & 199 & 49 \\
\hline 16 & 1995 & 37.4 & 45 & -92 & -239 & $107 \mathrm{I}$ \\
\hline 17 & 1995 & 45.1 & -186 & 105 & -126 & 45 \\
\hline 18 & 1995 & 46.7 & 179 & -93 & -108 & 57 \\
\hline 19 & 1995 & 40.2 & -37 & -71 & 160 & 128 \\
\hline 20 & 1995 & 45.9 & -161 & 87 & -337 & 49 \\
\hline 21 & 1995 & 36.3 & 46 & 150 & 107 & 100 \\
\hline 22 & 1995 & 37.6 & -73 & 107 & -257 & 129 \\
\hline
\end{tabular}


TABLE II. A list of all backgrounds with the expected number of background events in the $36<E_{e}<60 \mathrm{MeV}$ energy range for $R \geq 0$ and $R>30$. The neutrinos are from either $\pi$ and $\mu$ decay at rest (DAR) or decay in flight (DIF). Also shown are the number of events expected for $100 \% \bar{\nu}_{\mu} \rightarrow \bar{\nu}_{e}$ transmutation.

\begin{tabular}{|c|c|c|c|}
\hline Background & Neutrino Source & Events with $R \geq 0$ & Events with $R>30$ \\
\hline Beam Off & & $146.5 \pm 3.2$ & $2.52 \pm 0.42$ \\
\hline Beam-Related Neutrons & & $<0.7$ & $<0.1$ \\
\hline $\bar{\nu}_{\mathrm{e}} p \rightarrow e^{+} n$ & $\mu^{-} \rightarrow e^{-} \nu_{\mu} \bar{\nu}_{e}$ DAR & $4.8 \pm 1.0$ & $1.10 \pm 0.22$ \\
\hline $\bar{\nu}_{\mu} p \rightarrow \mu^{+} n$ & $\pi^{-} \rightarrow \mu^{-} \bar{\nu}_{\mu} \mathrm{DIF}$ & $2.7 \pm 1.3$ & $0.62 \pm 0.31$ \\
\hline $\bar{\nu}_{e} p \rightarrow e^{+} n$ & $\pi \rightarrow e \nu$ and $\mu \rightarrow e \nu \bar{\nu}$ DIF & $0.1 \pm 0.1$ & 0 \\
\hline Total with Neutrons & & $7.6 \pm 1.6$ & $1.72 \pm 0.38$ \\
\hline$\nu_{\mu} \mathrm{C} \rightarrow \mu^{-} X$ & $\pi^{+} \rightarrow \mu^{+} \nu_{\mu} \mathrm{DIF}$ & $8.1 \pm 4.0$ & $0.05 \pm 0.02$ \\
\hline$\nu_{\mathrm{e}}{ }^{12} C \rightarrow e^{-12} N$ & $\mu^{+} \rightarrow e^{+} \bar{\nu}_{\mu} \nu_{e}$ DAR & $20.1 \pm 4.0$ & $0.12 \pm 0.02$ \\
\hline$\nu_{e}{ }^{13} C \rightarrow e^{-13} N$ & $\mu^{+} \rightarrow e^{+} \bar{\nu}_{\mu} \nu_{e} \mathrm{DAR}$ & $22.5 \pm 4.5$ & $0.14 \pm 0.03$ \\
\hline$\nu e \rightarrow \nu e$ & $\mu^{+} \rightarrow e^{+} \bar{\nu}_{\mu} \nu_{e} \mathrm{DAR}$ & $12.0 \pm 1.2$ & $0.07 \pm 0.01$ \\
\hline$\nu e \rightarrow \nu e$ & $\pi \rightarrow \mu \nu_{\mu} \mathrm{DIF}$ & $1.5 \pm 0.3$ & $0.01 \pm 0.01$ \\
\hline$\nu_{e} \mathrm{C} \rightarrow e^{-} X$ & $\pi \rightarrow e \nu_{e} \mathrm{DAR}$ & $3.6 \pm 0.7$ & $0.02 \pm 0.01$ \\
\hline$\nu_{\mu} \mathrm{C} \rightarrow \pi X$ & $\pi \rightarrow \mu \nu_{\mu} \mathrm{DIF}$ & $0.2 \pm 0.1$ & 0 \\
\hline$\nu_{e} \mathrm{C} \rightarrow e^{-} X$ & $\pi \rightarrow e \nu$ and $\mu \rightarrow e \nu \bar{\nu}$ DIF & $0.6 \pm 0.1$ & 0 \\
\hline Total without Neutrons & & $68.6 \pm 7.4$ & $0.41 \pm 0.04$ \\
\hline Grand Total & & $222.7 \pm 8.2$ & $4.65 \pm 0.57$ \\
\hline 100\% Transmutation & $\mu^{+} \rightarrow e^{+} \bar{\nu}_{\mu} \nu_{e}$ DAR & $12500 \pm 1250$ & $2875 \pm 345$ \\
\hline
\end{tabular}



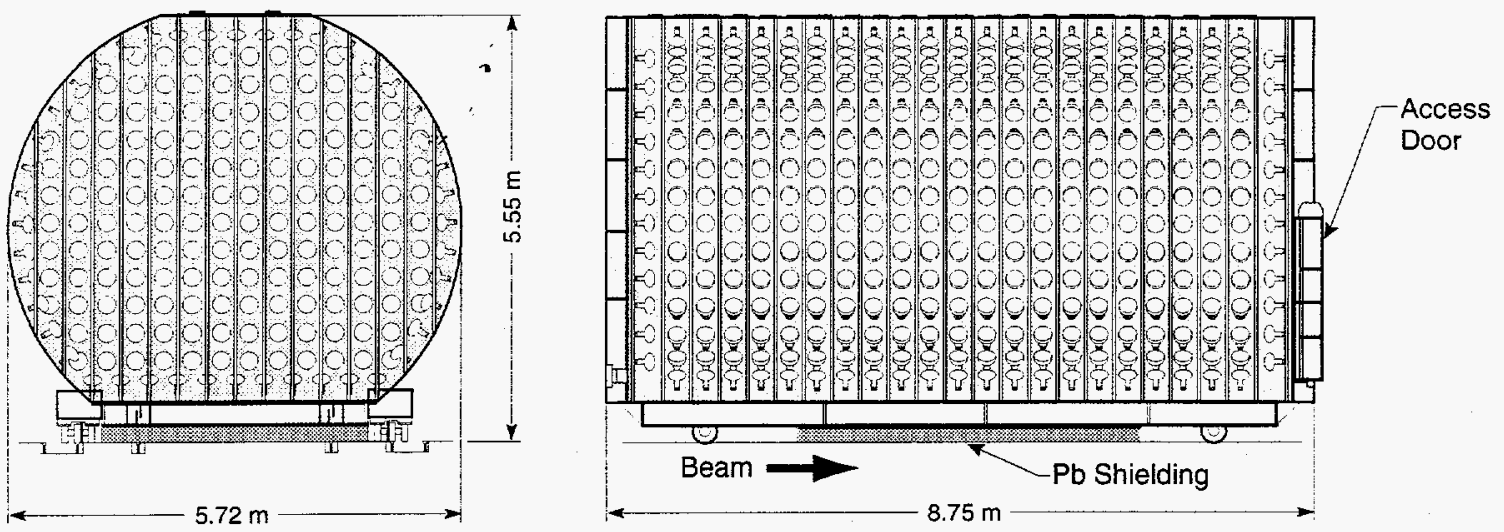

FIG. 1. A schematic of the LSND detector.

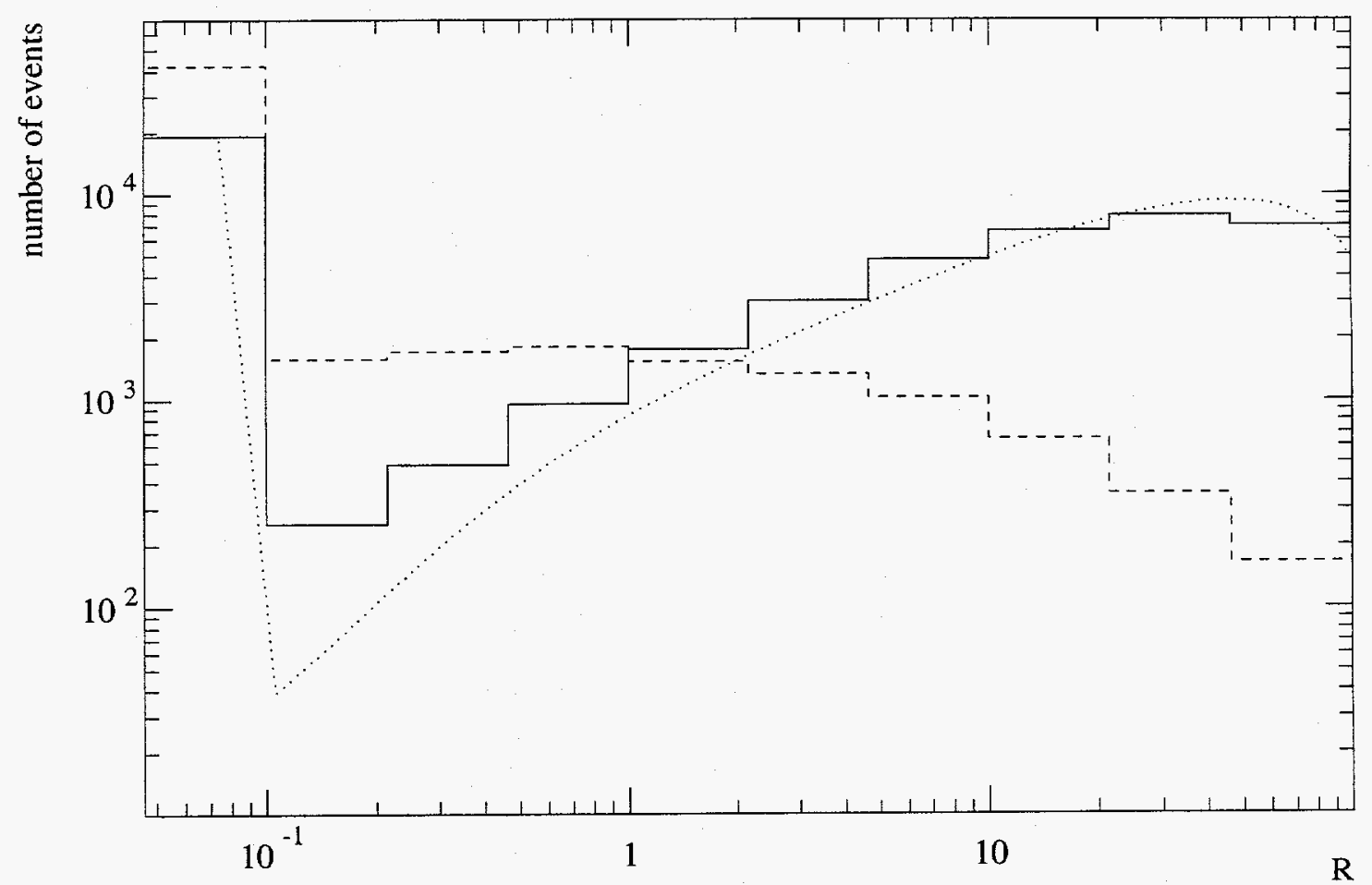

FIG. 2. Measured R distribution for events with the $\gamma$ correlated (solid) and uncorrelated (dashed) with the primary event. The dotted curve is the Monte Carlo $\mathrm{R}$ distribution for events with the $\gamma$ correlated. 


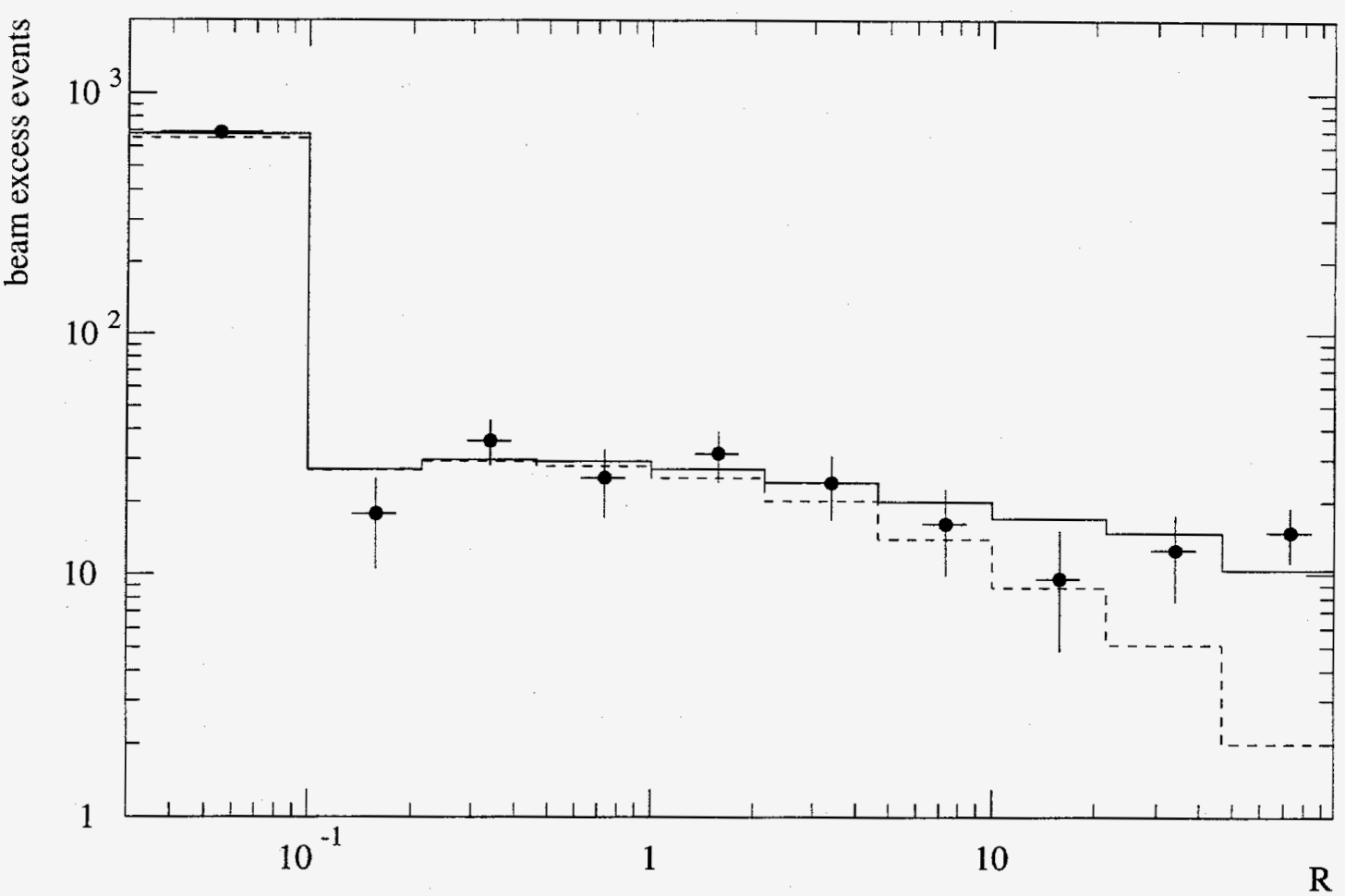

FIG. 3. The $\mathrm{R}$ distribution, beam on minus beam off excess, for events that have energies in the range $20<E_{e}<60 \mathrm{MeV}$. The solid curve is the best fit to the data, while the dashed curve is the component of the fit with an uncorrelated $\gamma$. 

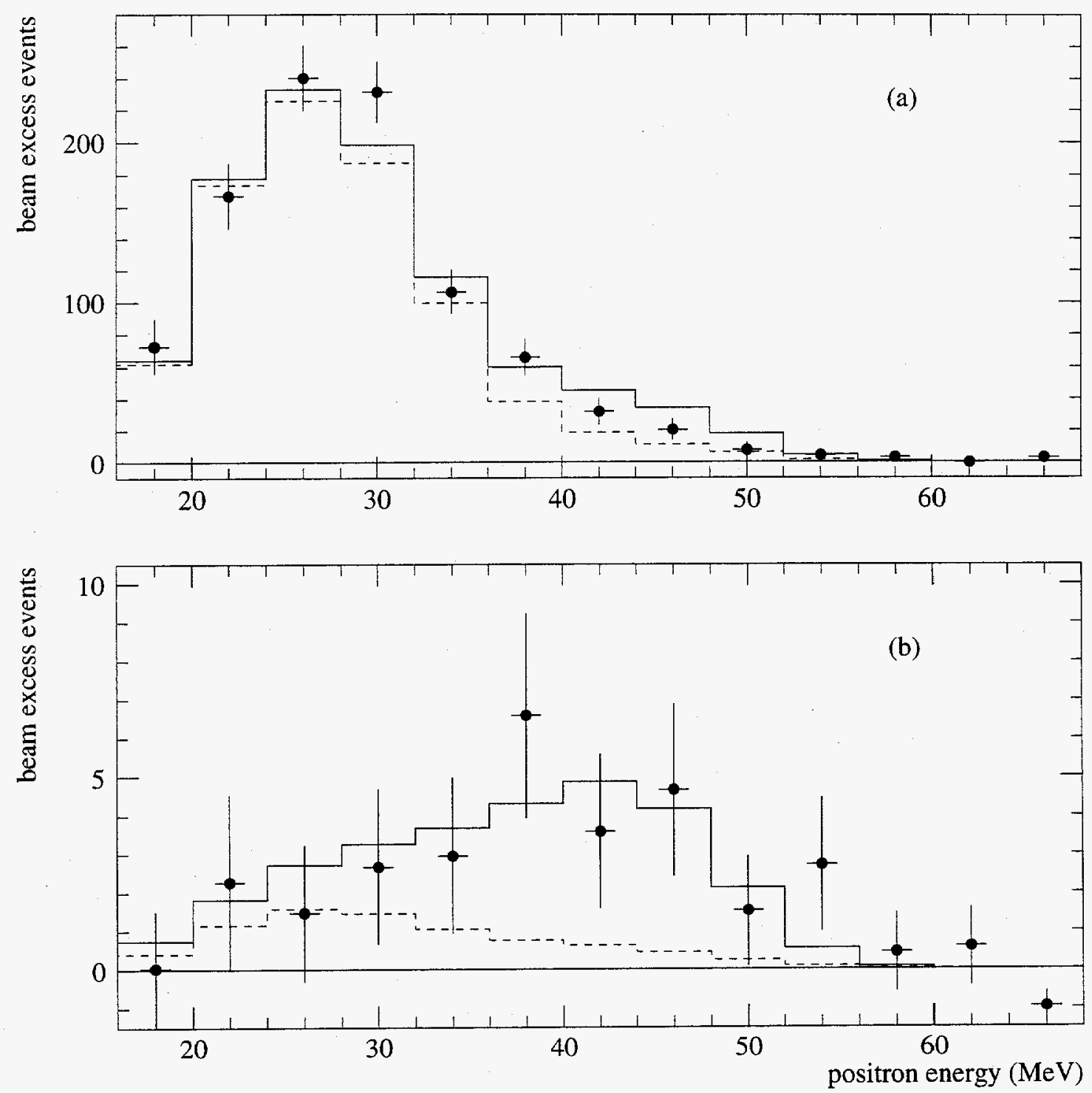

FIG. 4. The energy distribution for events with (a) $R \geq 0$ and (b) $R>30$. Shown in the figure are the beam-excess data, estimated neutrino background (dashed), and expected distribution for neutrino oscillations at large $\Delta m^{2}$ plus estimated neutrino background (solid). 

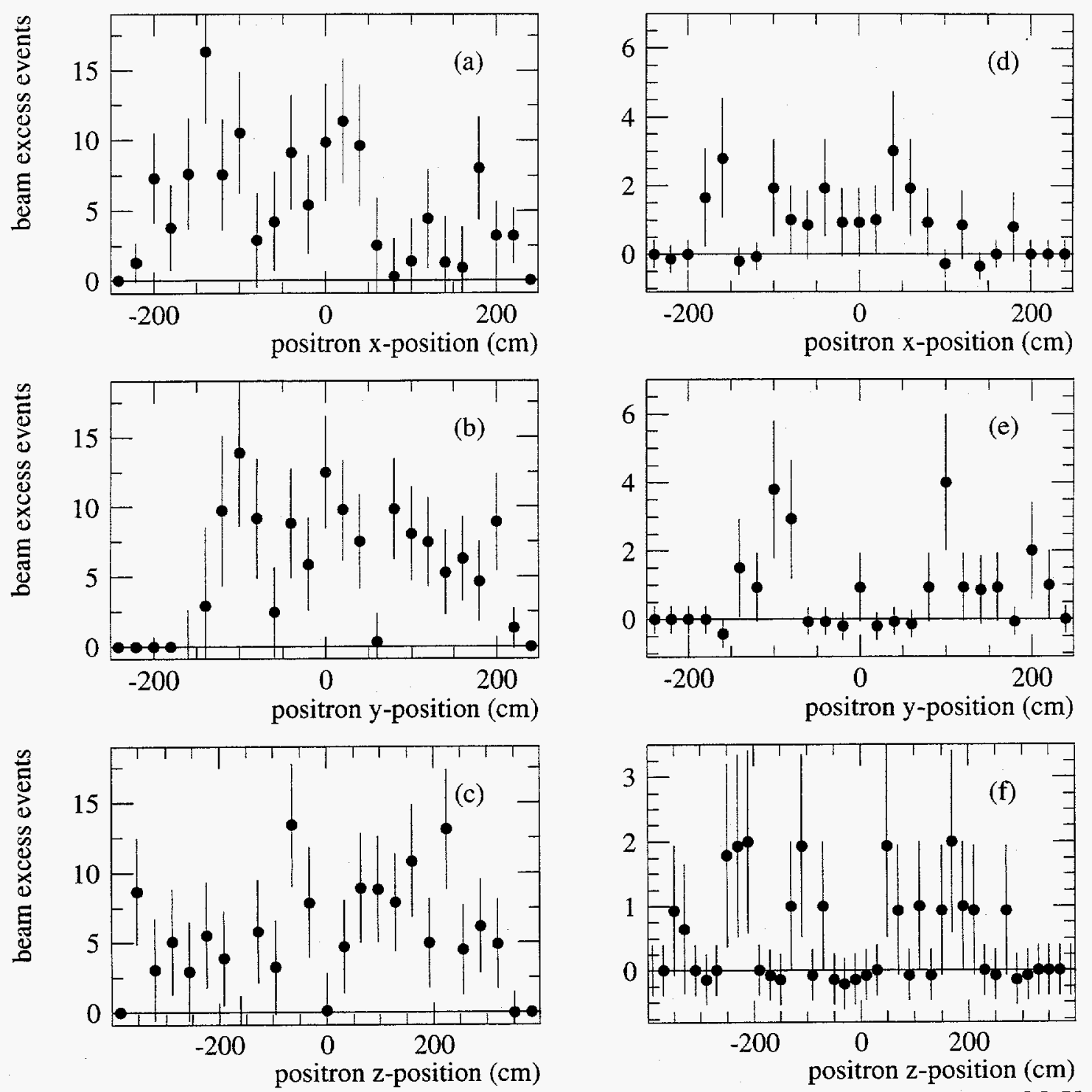

FIG. 5. The spatial distributions for beam-excess data events with $36<E_{e}<60 \mathrm{MeV}$. (a) -

(c) are for $R \geq 0$ and (d) - (f) are for $R>30$. 


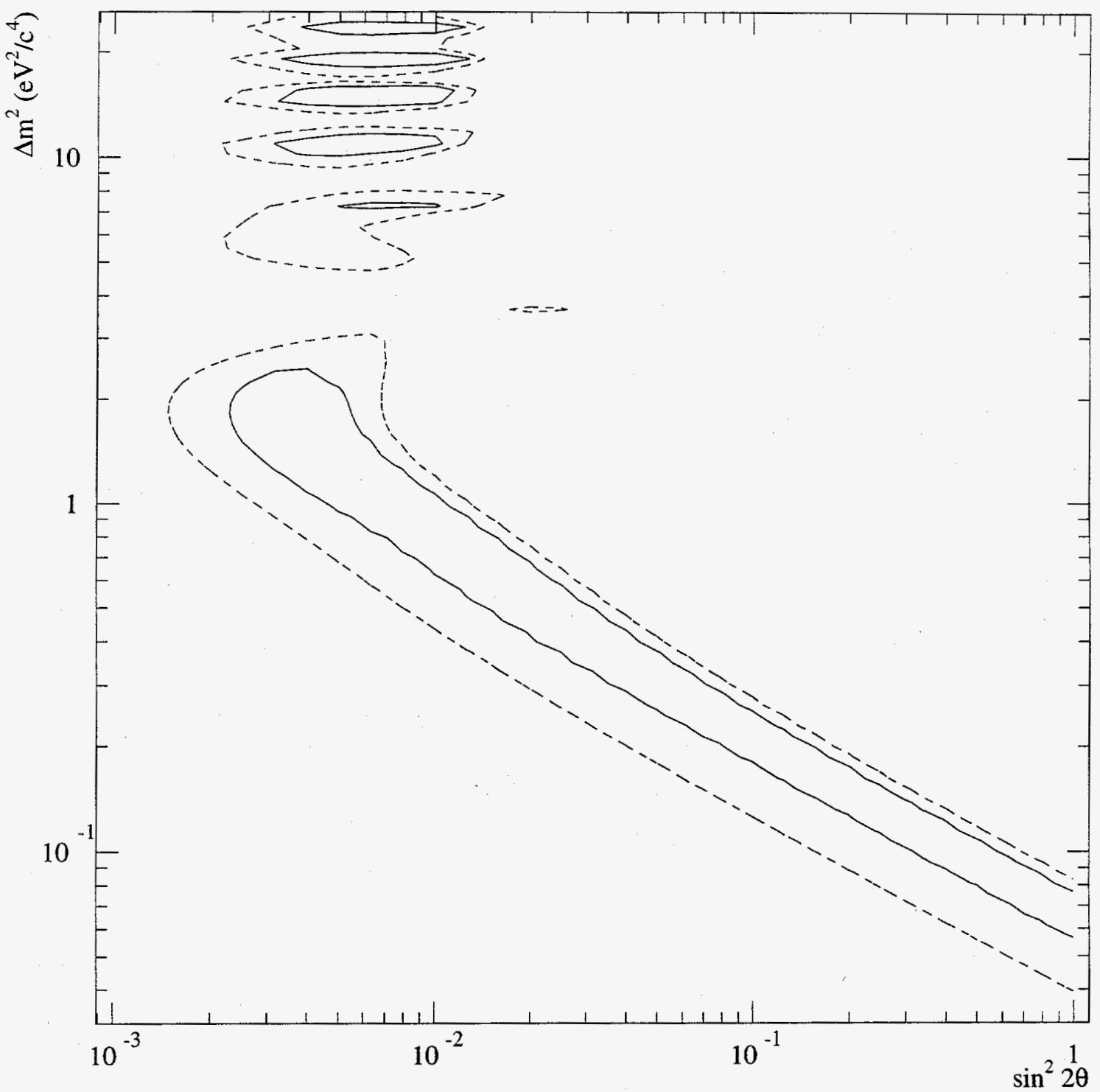

FIG. 6. Plot of the LSND $\Delta m^{2}$ vs $\sin ^{2} 2 \theta$ favored regions. They correspond to $90 \%$ and $99 \%$ likelihood regions after the inclusion of the effects of systematic errors. 